\title{
Constraining the strangeness content of the nucleon by measuring the $\phi$ meson mass shift in nuclear matter
}

\author{
Philipp Gubler 1 , 因 and Keisuke Ohtani ${ }^{2}$, 守 \\ ${ }^{1}$ RIKEN, Nishina Center, Hirosawa 2-1, Wako, Saitama, 351-0198, Japan \\ ${ }^{2}$ Tokyo Institute of Technology, Meguro 2-12-1, Tokyo 152-8551, Japan
}

(Dated: October 5, 2018)

\begin{abstract}
The behavior of the $\phi$ meson at finite density is studied, making use of a QCD sum rule approach in combination with the maximum entropy method. It is demonstrated that a possible mass shift of the $\phi$ in nuclear matter is strongly correlated to the strangeness content of the nucleon, which is proportional to the strange sigma term, $\sigma_{s N}=m_{s}\langle N|\bar{s} s| N\rangle$. Our results furthermore show that, depending on the value of $\sigma_{s N}$, the $\phi$ meson could receive both a positive or negative mass shift at nuclear matter density. We find that these results depend only weakly on potential modifications of the width of the $\phi$ peak and on assumptions made on the behavior of four-quark condensates at finite density. To check the stability of our findings, we take into account several higher order corrections to the operator product expansion, including $\alpha_{s}$-corrections, terms of higher order in the strange quark mass and terms of higher twist that have not been considered in earlier works.
\end{abstract}

\section{INTRODUCTION}

The strangeness content of the nucleon, $\langle N|\bar{s} s| N\rangle$ is an important quantity, both for understanding the effects of strange quarks on the nucleon structure [1] and the behavior of the strange quark condensate in dense matter. The second point is related to the fact that, via the Feynman-Hellmann theorem, the value of $\langle N|\bar{s} s| N\rangle$ determines, to leading order in density $\rho$, the pace of restoration of chiral symmetry of the strange quark sector in nuclear matter:

$$
\langle\bar{s} s\rangle_{\rho}=\langle\bar{s} s\rangle_{0}+\langle N|\bar{s} s| N\rangle \rho .
$$

The strange quark content of the nucleon also has implications that go beyond the physics of hadrons and the strong interaction, as it appears in the spin-independent elastic scattering cross sections of potential dark matter particles with nucleons and is one of the main sources of uncertainty for these cross sections [2, 3], therefore strongly affecting experimental dark matter searches. Hence, it is crucial to determine this quantity with high precision.

A variety of variables have been introduced in the literature to parametrize $\langle N|\bar{s} s| N\rangle$. We will in this paper mainly use

$$
\sigma_{s N}=m_{s}\langle N|\bar{s} s| N\rangle,
$$

and refer to it as the strange sigma term. This is a convenient quantity, because it is a renormalization group invariant, which is only related to the strange quark and does not directly depend on parameters related to $u$ and $d$ quarks, which have their own uncertainties. 11

In recent years, it has become possible to evaluate $\sigma_{S N}$ by direct lattice QCD calculations or by chirally extrapolating the

* pgubler@ riken.jp

† ohtani.k@th.phys.titech.ac.jp

1 Another commonly used variable is $y=\frac{\langle N|\bar{s}| N\rangle}{\langle N|\bar{q} q| N\rangle}=\frac{2 m_{q}}{m_{s}} \frac{\sigma_{s N}}{\sigma_{\pi N}}$, where $\langle N|\bar{q} q| N\rangle$ and $m_{q}$ are averaged quantities over the $u$ and $d$ quarks. $\sigma_{\pi N}$ stands for the $\pi N$ sigma term and is defined as $\sigma_{\pi N}=2 m_{q}\langle N|\bar{q} q| N\rangle$. Other parametrizations use $\sigma_{0}=(1-y) \sigma_{\pi N}$ or $f_{T_{s}}=\sigma_{s N} / m_{N}$, in which $m_{N}$ represents the nucleon mass. available lattice data [4-20]. These studies have shown that the value of $\sigma_{S N}$ is about a factor of 5 smaller than what was believed to be the correct range of values until about a decade ago. The values reported by the various groups however still show quite a large spread, lying roughly in the range of $0 \sim$ $70 \mathrm{MeV}$, which indicates that these calculations still contain systematic uncertainties.

We will in this paper discuss a different method for obtaining $\sigma_{s N}$, namely by experimentally measuring the mass of the $\phi$ meson in nuclear matter. The behavior of the $\phi$ meson as a function of density strongly depends on the value of $\sigma_{S N}$ and can therefore provide a strong constraint for this quantity once it is measured with sufficiently high precision. The relation between the $\phi$ meson mass and $\sigma_{s N}$ [shown in Fig. 4 ] and Eq. (13)] is derived from a recently developed method combining QCD sum rules with the maximum entropy method (MEM) [21]. This method allows us to extract the most probable spectral function directly from the sum rules, without having to make strong assumptions on its functional form. Moreover, by making use of MEM, it is possible to determine the mass shift of the $\phi$ meson quite precisely. As shown in Sec. III A, we have tested the reproducibility of the mass shift in a series of mock data tests, in which we have determined the precision of the mass shift extraction by MEM to be of the order of $5 \mathrm{MeV}$, which is good enough for our purposes.

QCD sum rule studies of light vector mesons at finite density in fact already have quite a long history [22-32]. They have especially attracted much interest because QCD sum rules provide relations between the partial restoration of chiral symmetry in nuclear matter and modifications of meson spectra that could be measured in experiments [33, 34]. The early works on this subject have usually focused on the $\rho$ and $\omega$ channels and the relation between their mass shifts and chiral symmetry. By now, it is however understood that this relation is not a simple one, as the driving term for the modification of the spectrum entering into the sum rules contains not the most simple two-quark condensate but the more involved four-quark condensate. Moreover, for the $\rho$ meson, the spectral modification cannot be assumed to be a simple mass shift of the ground state peak, but is rather a combination of mass 
shift and broadening for which the sum rules only provide a relatively weak constraint [28].

These issues are less severe for the $\phi$ meson. Due to the effects of the strange quark mass on the operator product expansion (OPE), it is for the $\phi$ meson channel mainly the dimension-four term that governs the modification of the spectral function and the dimension-six term (in which the fourquark condensate dominates) is merely a small correction with no large effects. As it has already been pointed out in earlier works [22, 27, 31], this leads to an unambiguous relation between the $\phi$ meson mass shift and the strange sigma term, which appears at dimension four. Furthermore, even though the $\phi$ is expected to experience some broadening when put into nuclear matter, it is known that its width will not grow above the $100 \mathrm{MeV}$ level and might even stay much below, namely around $75 \mathrm{MeV}$ [35] or at an even smaller value [36]. Therefore, the $\phi$ meson will retain its character as a relatively narrow peak in the spectral function that facilitates its analysis as no severe complications from large broadening effects arise.

Because of these advantages, one can expect that the predictive power of the sum rules of the $\phi$ is bigger than for the $\rho$ and $\omega$. To check whether this expectation is actually true and to quantify in what way the sum rules constrain the properties of the $\phi$ meson peak, and, thus, inversely, in what way the $\phi$ meson mass constrains the values of $\sigma_{s N}$, is the main goal of this paper.

In Sec. $\Pi \mathrm{A}$ we will first recapitulate the basics of QCD sum rules and their application to finite density. Next, in Sec. IIB, the OPE of the $\phi$ meson channel in vacuum and at finite density will be given. Then, to demonstrate the ability of MEM to reproduce mass shifts at finite density, the results of our mock data analyses will be summarized in Sec. IIIA. Finally, the analysis results of the OPE data are provided and discussed in Secs. [IIB and IV] while the conclusions are given in Sec. D

\section{FORMALISM}

\section{A. Basics of QCD sum rules}

As usual when working with QCD sum rules [37], we start with the two-point function of an interpolating field coupling strongly to the $\phi$ meson:

$$
\Pi_{\mu v}(\omega, \vec{q})=i \int d x^{4} e^{i q x}\left\langle\mathrm{~T}\left[j_{\mu}(x) j_{v}(0)\right]\right\rangle_{\rho} .
$$

Here, the operator $j_{\mu}(x)$ is defined as $j_{\mu}(x)=\bar{s}(x) \gamma_{\mu} s(x)$, and \langle\rangle$_{\rho}$ stands for the expectation value with respect to the ground state of nuclear matter at $T=0$. Generally, $\Pi_{\mu v}(\omega, \vec{q})$ contains two independent Lorentz structures [38], but for the case of the $\phi$ meson at rest relative to the nuclear medium $(\vec{q}=0)$, there is only one such structure and it suffices to consider the contracted correlator defined as $\Pi\left(\omega^{2}\right)=-\frac{1}{3 \omega^{2}} \Pi_{\mu}^{\mu}(\omega, \vec{q}=0)$. From the analyticity of $\Pi\left(\omega^{2}\right)$, the dispersion relation

$$
\Pi\left(\omega^{2}\right)=\frac{1}{\pi} \int_{0}^{\infty} d s \frac{\operatorname{Im} \Pi(s)}{s-\omega^{2}-i \varepsilon}
$$

can be derived. The idea of the QCD sum rule approach is now to take $\omega^{2}$ as a large and negative number and to calculate the left-hand side of Eq.(4) using the OPE. This results in a power series in $1 / \omega^{2}$ with (Wilson) coefficients expressed as expansions in the strong coupling constant $\alpha_{s}$. On the right hand side, the function $\frac{1}{\pi} \operatorname{Im} \Pi(s)$ is viewed in terms of hadronic degrees of freedom that couple to the operator $j_{\mu}(x)$.

Equation (4) is in fact not yet the final form of the sum rule as the integral on its right-hand side is not convergent and thus a subtraction term is needed. The standard way to remedy this problem is the application of the Borel transform, which cancels any subtraction constant, renders the integral over $s$ convergent and furthermore improves the convergence of the OPE. This finally gives

$$
\Pi\left(M^{2}\right)=\frac{2}{M^{2}} \int_{0}^{\infty} d \omega e^{-\omega^{2} / M^{2}} \omega A(\omega),
$$

where we have defined the spectral function $A(\omega)$ as $A(\omega)=$ $\frac{1}{\pi} \operatorname{Im} \Pi\left(\omega^{2}\right)$. This is the final form of the sum rule that will be used in the analyses presented in this paper.

\section{B. OPE of the $\phi$ meson channel in vacuum and at finite density}

The result of the OPE is generally obtained as shown below:

$$
\Pi_{\mathrm{OPE}}\left(M^{2}, \rho\right)=c_{0}(\rho)+\frac{c_{2}(\rho)}{M^{2}}+\frac{c_{4}(\rho)}{M^{4}}+\frac{c_{6}(\rho)}{M^{6}}+\ldots
$$

In this work, we consider terms up to dimension six. The coefficients $c_{i}(\rho)$ have mass dimension $i$ and can contain logarithmic dependencies on the Borel mass $M$, which we have not explicitly spelled out in Eq. (6) for simplicity of notation.

The coefficients in the vacuum $(\rho=0)$ can be given as follows [37, 39-42]:

$$
c_{0}(0)=\frac{1}{4 \pi^{2}}\left(1+\frac{\alpha_{s}}{\pi}\right), \quad c_{2}(0)=\frac{m_{s}^{2}}{4 \pi^{2}}\left[-6-4 \frac{\alpha_{s}}{\pi}\left(4-6 \log \left(\frac{M}{\mu}\right)+3 \gamma_{E}\right)\right]
$$




$$
\begin{aligned}
c_{4}(0)= & \frac{1}{12}\left(1+\frac{7}{6} \frac{\alpha_{s}}{\pi}\right)\left\langle\frac{\alpha_{s}}{\pi} G^{2}\right\rangle+2 m_{s}\left(1+\frac{1}{3} \frac{\alpha_{s}}{\pi}\right)\langle\bar{s} s\rangle+\frac{3}{4 \pi^{2}} m_{s}^{4}\left[1+4 \log \left(\frac{M}{\mu}\right)-2 \gamma_{E}\right] \\
& -\frac{1}{6 \pi^{2}} m_{s}^{4} \frac{\alpha_{s}}{\pi}\left[35-3 \pi^{2}-24 \zeta(3)+3\left(2 \log \left(\frac{M}{\mu}\right)-\gamma_{E}\right)+18\left(2 \log \left(\frac{M}{\mu}\right)-\gamma_{E}\right)^{2}\right], \\
c_{6}(0)= & -\frac{112}{81} \pi \alpha_{s} \kappa_{0}\langle\bar{s} s\rangle^{2}+\frac{1}{18} m_{s}^{2}\left\langle\frac{\alpha_{s}}{\pi} G^{2}\right\rangle-\frac{4}{3} m_{s}^{3}\langle\bar{s} s\rangle .
\end{aligned}
$$

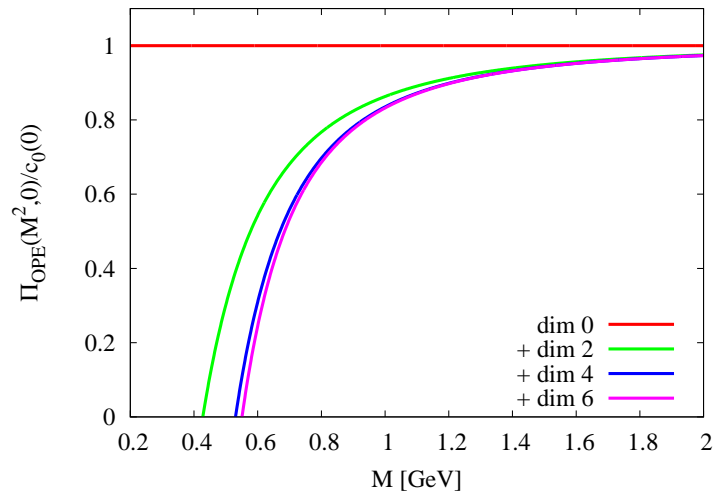

FIG. 1. The OPE terms in vacuum, given in Eqs.7.9, as a function of the Borel mass $M$. The contributions are shown relative to the leading order OPE term, $c_{0}(0)$.

Up to dimension four, we have taken into account the first order $\alpha_{s}$ corrections to all the Wilson coefficients, which were calculated already a long time ago [40, 41], but have to our knowledge not been taken into account in the available sum rule studies on the $\phi$ meson. These corrections, however, turn out to be quite small, namely around $10 \%$ of the leading order terms or smaller, with the exception of the $m_{s}^{2}$ and $m_{s}^{4}$ terms. Note that for full consistency, we, in principle, should have included the terms of $\alpha_{s}^{2}$ in the perturbative dimension-zero term of Eq. (7), since they correspond to the hard parts of the diagrams that give the first order $\alpha_{s}$ corrections to the Wilson coefficients of the gluon condensate, which we have taken into account in Eq.8). As these $\alpha_{s}^{2}$ terms, in contrast to their counterparts appearing with the gluon condensate, however do not give any contribution to the density dependence of the OPE and hence to the $\phi$ meson mass shift, we have ignored them here.

Furthermore, we include several higher order terms in the strange quark mass $m_{s}$, which have not been considered before: $m_{s}^{4}$ [40], $m_{s}^{2}\left\langle\frac{\alpha_{s}}{\pi} G^{2}\right\rangle$ and $m_{s}^{3}\langle\bar{s} s\rangle$ [39]. These corrections have also turned out to be small compared to other terms of the same dimension. Note that there are in fact more condensates of dimension six, that can, in principle, appear in the OPE. Specifically, these are $m_{s}\langle\bar{s} g \sigma \cdot G s\rangle$ and $\left\langle g^{3} G^{3}\right\rangle$, whose Wilson coefficients, however, are known to vanish at leading order in $\alpha_{s}$ [39]. One further point worth mentioning here is concerned with the four-quark condensate term at dimension six. To obtain its form given in Eq. (9) we have assumed that the vacuum saturation approximation holds, and have parametrized the possible breaking of this approximation by the parameter $\kappa_{0}$. In vacuum we will assume that $\kappa_{0}$ is 1 (and thus that the vacuum saturation approximation is exact), but will consider its deviation from 1 later in the finite density case.

To get an idea of the behavior of the various terms of Eqs. (7. 9), they are plotted in Fig. 1. For drawing this plot, we have used $\alpha_{s}=0.5[43],\left\langle\frac{\alpha_{s}}{\pi} G^{2}\right\rangle=0.012 \pm 0.0036 \mathrm{GeV}^{4}$ [44], $m_{s}=128 \pm 7 \mathrm{MeV}$ [45], $\langle\bar{s} s\rangle=(0.6 \pm 0.1)\langle\bar{q} q\rangle$ [46], $\langle\bar{q} q\rangle=-(0.232 \pm 0.06)^{3} \mathrm{GeV}^{3}$ [47], and $\kappa_{0}=1$. All these values are given at renormalization scale $1 \mathrm{GeV}$.

It is observed in Fig. 1 that the qualitative properties of the OPE are essentially determined by the first three terms while the fourth term, which is dominated by the four-quark condensate, is only a small correction. This is fortunate, as the four-quark condensate is not well known, which in the parametrization used here translates into our lack of knowledge of the actual value of $\kappa_{0}$.

Next, for investigating the finite density case, we need to calculate the $\rho$ dependence of the coefficients $c_{i}(\rho)$ in Eq. (6). To obtain $c_{i}(\rho)$ for general values of $\rho$ is a very difficult task that is beyond our ability at the present time. What we, however, can do, is to restrict ourselves to low densities and assume that the linear density approximation is valid for the density regime that we are interested in. We are in this work mainly interested in the modification of the $\phi$ meson at nuclear matter density and there is evidence that this approximation indeed works well there [22, 26]. We will further discuss this point in Sec. IV and for the moment just assume that we are considering densities at which the above assumption is valid. The $\rho$ dependence of the coefficients $c_{i}(\rho)$ at linear order has already been discussed many times in the literature [22, 27, 30]; the main focus, however, was usually laid on the $\rho$ and $\omega$ channels. We here give the result for the $\phi$ channel, which again includes several new terms that have not been taken into account in the works published so far:

$$
c_{0}(\rho)=c_{0}(0), \quad c_{2}(\rho)=c_{2}(0),
$$




$$
\begin{aligned}
c_{4}(\rho)= & c_{4}(0)+\rho\left[-\frac{2}{27}\left(1+\frac{7}{6} \frac{\alpha_{s}}{\pi}\right) M_{N}+\frac{56}{27} m_{s}\left(1+\frac{61}{168} \frac{\alpha_{s}}{\pi}\right)\langle N|\bar{s} s| N\rangle\right. \\
& \left.+\frac{4}{27} m_{q}\left(1+\frac{7}{6} \frac{\alpha_{s}}{\pi}\right)\langle N|\bar{q} q| N\rangle+\left(1-\frac{5}{9} \frac{\alpha_{s}}{\pi}\right) A_{2}^{s} M_{N}-\frac{7}{12} \frac{\alpha_{s}}{\pi} A_{2}^{g} M_{N}\right] \\
c_{6}(\rho)= & c_{6}(0)+\rho\left[-\frac{224}{81} \pi \alpha_{s} \kappa_{N}\langle\bar{s} s\rangle\langle N|\bar{s} s| N\rangle-\frac{104}{81} m_{s}^{3}\langle N|\bar{s} s| N\rangle\right. \\
& \left.+\frac{8}{81} m_{s}^{2} m_{q}\langle N|\bar{q} q| N\rangle-\frac{4}{81} m_{s}^{2} M_{N}-\frac{3}{4} m_{s}^{2} A_{2}^{s} M_{N}-\frac{5}{6} A_{4}^{s} M_{N}^{3}\right] .
\end{aligned}
$$

The novel terms here are the $\alpha_{s}$ corrections of the terms at dimension four, a term related to a twist- 2 gluonic operator at dimension four and several terms of higher order in $m_{s}$ at dimension six. Most of these terms only have a small effect and do not much change the earlier results. The only exception is the twist-2 gluonic operator at dimension four, which is proportional to the first moment of the gluonic parton distribution $A_{2}^{g}$. This term is in fact almost as large as the twist-2 strange quark operator (proportional to $A_{2}^{s}$ ), and therefore cannot be ignored. The definitions of $A_{2}^{s}, A_{4}^{s}$ and $A_{2}^{g}$, which are all moments over parton distribution functions, can be found for instance in [50].

For the numerical evaluation of Eqs. 10, 12), we use $M_{N}=$ $940 \mathrm{MeV}, 2 m_{q}\langle N|\bar{q} q| N\rangle=45 \pm 7 \mathrm{MeV}$ [49], $A_{2}^{s}=0.044 \pm$ $0.011, A_{4}^{s}=0.0011 \pm 0.0004$, and $A_{2}^{g}=0.359 \pm 0.146$. The last three values have been extracted numerically from the parton distributions given in [51]. The breaking of the factorization assumption of the four-quark condensates is parametrized using $\kappa_{N}$. Here, we follow the treatment of [31] and take into account the possibility that $\kappa_{N}$ can differ from the vacuum value $\kappa_{0}$. Specifically, we will consider the range $\kappa_{N}=$ $1 \pm 1$. Note that we have not explicitly included twist- 4 terms, which, in principle, can appear at dimension six. In [26, 48], the magnitude of these terms has been estimated for the $\rho(\omega)$ meson case to be 1.36 (2.29) times the corresponding twist2 contribution of the same dimension. For the calculation of this paper, we assume that the ratio between twist- 2 and twist4 terms has the same order of magnitude for the $\phi$ meson case and take the average value of 1.83 for this ratio. It is clear that this only a very crude estimate and we therefore will attach to it an uncertainty of $100 \%$ and use $1.83 \pm 1.83$ when computing the error of the final results. Our poor knowledge of the twist- 4 contribution does, however, not cause serious problems, as its contribution is negligibly small. Increasing for instance the above ratio by a factor of 2 only results in a mass shift of the $\phi$ meson peak of at most $1 \mathrm{MeV}$ at nuclear matter density. As explained in the introduction, we do not assume any value for the strange sigma term $m_{s}\langle N|\bar{s} s| N\rangle$, but treat it as a free parameter.

Let us here also make a few comments on the derivation of Eq.11). One might wonder where the term containing the (light quark) sigma term $2 m_{q}\langle N|\bar{q} q| N\rangle$ comes from, even though we are considering a correlator of an interpolating field constructed from only strange quarks. This term arises from the density dependence of the gluon condensate, which to leading order in $\rho$ is proportional to the nucleon mass in the chiral limit [52, 53]: $\left\langle\frac{\alpha_{s}}{\pi} G^{2}\right\rangle_{\rho}=\left\langle\frac{\alpha_{s}}{\pi} G^{2}\right\rangle_{\rho=0}-\frac{8}{9}\left(M_{N}-\sigma_{\pi N}-\right.$ $\left.\sigma_{s N}\right) \rho$.

Furthermore, it is also worth mentioning that the derivation of the twist- 2 gluonic operator term is somewhat nontrivial as it can only be systematically obtained by taking into account the concept on non-normal ordered operators and their expectation values with respect to the nuclear matter ground state, as only for these the OPE is generally well defined in the chiral limit [54]. This procedure corresponds to subtracting out the soft contributions of perturbative quark propagators with attached gluon lines. A detailed account of how the related actual calculations can be done has been given recently in [55] for the case of heavy-light quark pseudoscalar mesons and we here have followed the method put forward in that publication to obtain this twist-2 gluonic operator term of dimension four. For details, we thus refer the reader to [55] and the references cited therein. 2

\section{ANALYSIS RESULTS OF THE SUM RULES IN VACUUM AND FINITE DENSITY}

\section{A. MEM test analysis of mock data}

Before directly analyzing the OPE of the previous section, we here at first will discuss the results of some test analysis of artificial mock data. This sort of test is important for confirming what properties of the spectral function MEM is able to reproduce and thus for getting an idea on the systematic error of this approach. For details of MEM, we refer the reader to [57, 58], and for practical details specific to the application of MEM to QCD sum rules, to [21, 56].

First, we show the result of the mock data analysis of a spectral function resembling the one of the $\phi$ meson channel in the vacuum. For this, we use a realistic input spectral function, which has been fitted to experimental data in [59], create mock OPE data by substituting this spectral function

\footnotetext{
${ }^{2}$ Let us however state here that we could not reproduce one small part of the formulas given in [55]. Specifically, we got $\log \frac{\mu^{2}}{m_{q}^{2}}-\frac{1}{2}$ instead of $\log \frac{\mu^{2}}{m_{q}^{2}}-$ $\frac{1}{3}$ in a factor appearing in the last term of Eq.(56) of [55]. We will in this study use our own result, but note that this disagreement only has a very small numerical effect on the OPE.
} 


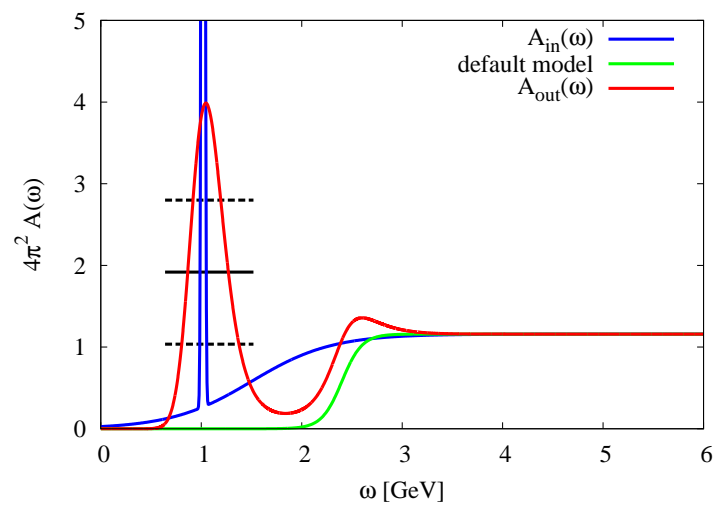

FIG. 2. Result of the MEM analysis of mock data $\left(A_{\text {out }}(\omega)\right)$ is plotted as a red line. The mock data were constructed from $A_{\text {in }}(\omega)$, which is shown by the blue line.

into the right-hand side of Eq. (5) and then analyze these mock data with MEM. The analyzed Borel mass region is taken to be the same as for the OPE data analysis of the following subsection. Specifically, we take $M_{\min }=0.56 \mathrm{GeV}$ for the lower boundary, which is determined from the convergence criterion of the OPE, which demands that the highest order OPE term should be smaller than $10 \%$ of the leading perturbative term of dimension zero. For the upper boundary, we use $M_{\max }=1.10 \mathrm{GeV}$. This rather small value for $M_{\max }$ is taken to suppress the continuum contributions to the sum rules. The result $A_{\text {out }}(\omega)$ is plotted in Fig. 2, in which the input spectral function $A_{\text {in }}(\omega)$ and the employed default model (which is an input of the MEM analysis [57, 58]) are also shown for comparison. We evaluate the peak position by taking the average of $\omega$ over the peak region, which is defined as the interval for which the spectral function takes values above half of the maximum peak height. We will use the same prescription for the rest of this paper. This then leads to a value lying $44 \mathrm{MeV}$ above the input peak position, which gives an idea of the quantitative accuracy of MEM for this particular quantity. We will, however, see below that when considering mass shifts, the precision will be considerably improved. It is furthermore understood from Fig. 2 that the output $\phi$ peak is strongly broadened due to artificial MEM effects, which indicates that obtaining meaningful information on the width of the $\phi$ meson at finite density from our MEM approach will be a rather difficult task. We thus in the following concentrate our efforts only on the mass shift.

To test the quantitative ability of MEM to reproduce such a mass shift and how a possible broadening of the peak will influence the result, we will as a next step study several kinds of mock data, which include mass shifted peaks with various degrees of broadening. A few representative results are shown in Fig. 3, where input mass shifts are compared with their MEM outputs. As one can observe from this plot, the mass shifts are mostly reproduced well, the error only being a few $\mathrm{MeV}$. It is however also seen that the mass shift is underestimated especially for large positive mass shifts. We will take this effect into account when extracting the $\phi$ meson mass shift from the

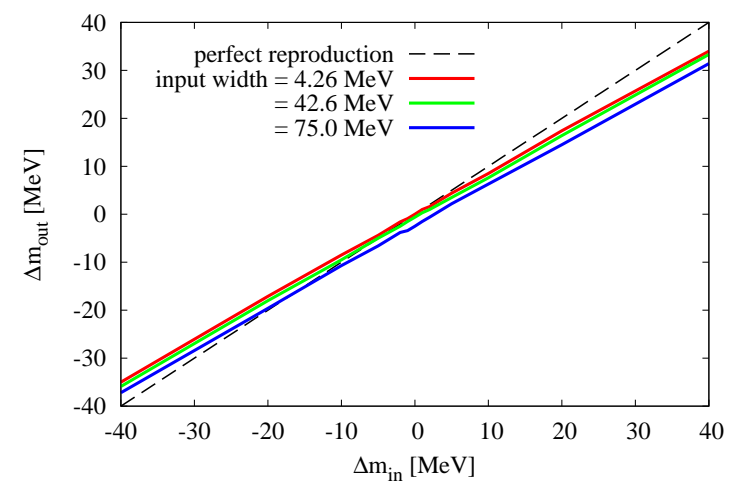

FIG. 3. Result of the MEM analysis of mock data with mass shifted peaks and various degrees of broadening. The mass shift obtained by the analysis is given as a function of the input mass shift of the mock data.

actual OPE data. We furthermore note that broadening somewhat decreases the MEM output mass, which introduces some uncertainty into our results.

We have also investigated the effect of a possible dependence of the continuum on the density. Even though we do not have any detailed knowledge on the behavior of the continuum in dense matter, we can make a crude estimate by assuming that it is dominated by freely propagating kaons with properties modified by the background density. It is known from effective models based on chiral symmetry that the kaons on the average receive a negative mass shift of below $50 \mathrm{MeV}$ at nuclear matter density [60, 61], which can be translated into a shift (of below $100 \mathrm{MeV}$ ) of the continuum towards smaller energies. We have also considered the possibility of the continuum becoming smoothed out due to density effects and have allowed the gradient of the continuum to decrease up to $10 \%$. Including these modifications into our analysis has again an effect of at most a few $\mathrm{MeV}$ on the peak position of the $\phi$ meson and hence leads to a further systematic uncertainty. Let us furthermore stress here that the assumptions on the behavior of the continuum mentioned above are only used to generate mock data for the MEM test analysis presented here. In the MEM analysis of the real OPE data of the next section, no assumptions are made on the actual behavior of the continuum.

All the effects discussed in the last two paragraphs will be taken into account when evaluating the error of our final results.

\section{B. MEM analysis of OPE data in vacuum and at finite density}

After the investigation of mock data of the last section, we are now in a position to study the actual OPE and to give an accurate interpretation of the obtained results.

Let us start with the spectral function in vacuum, for which we analyze the OPE data of Eqs.(7) 9 ). The result closely resembles the one of Fig. 2 and we thus do not show it here. For the peak position $\left(m_{\phi}\right)$, we get a value of $1.075 \mathrm{GeV}$, which 
lies $56 \mathrm{MeV}$ above the experimental value of $1.019 \mathrm{GeV}$. Note that we have deliberately chosen a rather small value for the strange quark condensate to get this mass. This is done in purpose of starting the analysis from a spectral function in the vacuum that is as realistic as possible, as higher quark condensate values would lead to an even larger $m_{\phi}$.

Next, we proceed to the main subject of interest of this paper, the behavior of the $\phi$ meson at finite density. As a first example, we choose two values of the strange sigma term, provided by recent lattice QCD calculations [10, 12], for which we have intentionally chosen results that lie on the lower and upper range of the values reported during the past few years. They will therefore provide a lower and upper limit for the mass shift of the $\phi$ meson, based on these lattice results. The behavior of the $\phi$ meson mass as a function of density is shown in the upper plot of Fig. 4, where it is seen that the $\phi$ meson mass shift at nuclear matter density lies roughly in the range of $+10 \mathrm{MeV} \sim-10 \mathrm{MeV}$.

This result is especially interesting in view of the fact that earlier sum rule studies have all [22- $25,27,31,32]$ obtained a negative mass shift at nuclear matter density, while here we get both the possibility of a positive and negative mass shift, depending on the value of $\sigma_{S N}$. The reason for this discrepancy is twofold. First, the recent lattice QCD values of the strange sigma term are much smaller than those that had been used until about a decade ago, which significantly reduces the contribution of this term to the OPE of Eq. (12). Furthermore, the twist-2 gluonic term of dimension four, which was not considered in these works, has turned out to have quite a large effect, leading to a further increase of the mass. Hence, the situation is now quite different from what it used to be and it is at present not even clear whether there will be a positive, negative or any mass shift at all at nuclear matter density.

In this context, let us mention the works using hadronic models with phenomenologically determined effective Lagrangians [27, 62-64], which at normal nuclear matter density get a small but negative mass shift of below $10 \mathrm{MeV}$ and a width about an order of magnitude larger than the vacuum value. As can be observed in Fig. 4, this is consistent with our QCD sum rule result and some of the recent lattice QCD computation ranges of $\sigma_{s N}$, but would exclude too-small values of the strange sigma term, for which the mass shift is positive.

As explained in the introduction, we do not choose any specific value of the strange sigma term, but study the modification of the $\phi$ meson more generally as a function of this parameter. The result of this investigation is given in the lower plot of Fig. 4 where the $\phi$ meson mass at nuclear matter density is shown as a function of $\sigma_{s N}$. Here, the error band includes the uncertainties of $A_{2}^{s}, A_{4}^{s}, A_{2}^{g}, 2 m_{q}\langle N|\bar{q} q| N\rangle, \kappa_{N}$, and of the twist- 4 terms of dimension six. Furthermore, the systematic errors of the MEM analysis stemming from the possible broadening of the $\phi$ meson peak and the modification of the continuum, discussed at the end of Sec. IIIA are also taken into account. Figure 4 clearly demonstrates that there is an (almost) linear relationship between the $\phi$ meson mass shift and $\sigma_{s N}$. Altogether, the result of Fig. 4 can most simply
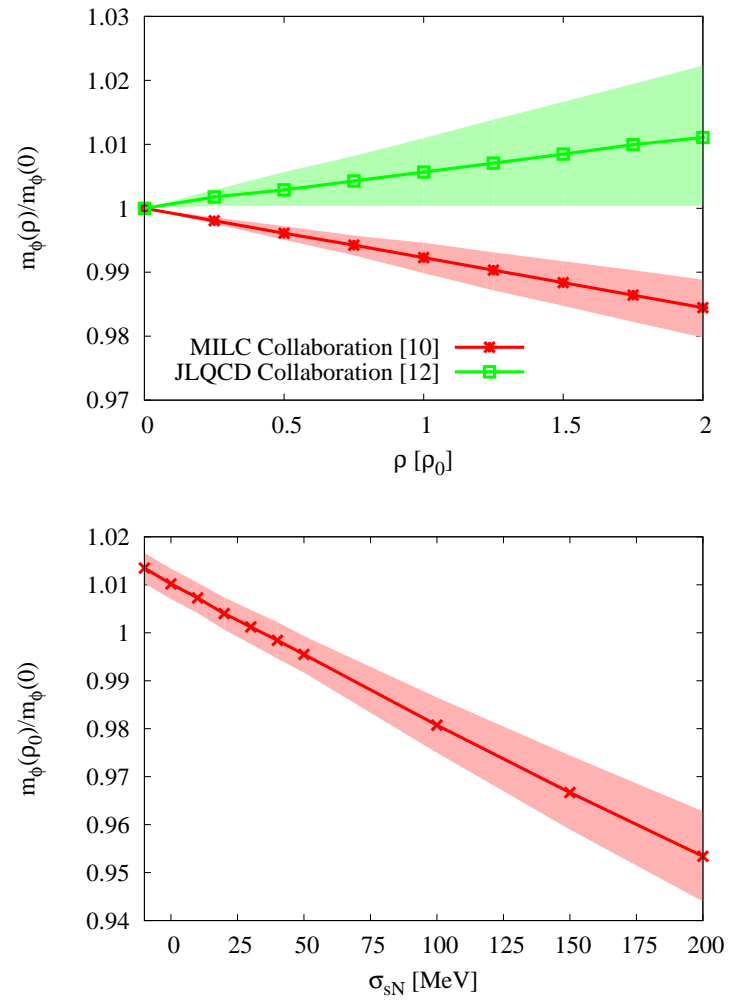

FIG. 4. (Upper plot) Peak position of $\phi$ meson as a function of the density $\rho$, for value ranges of the strange sigma term $\sigma_{s N}$, obtained from the MILC [10] and JLQCD [12] lattice QCD collaborations. The $\sigma_{s N}$ values are $61 \pm 9 \mathrm{MeV}$ for MILC and $8 \pm 21 \mathrm{MeV}$ for JLQCD. (Lower plot) Peak positions of the $\phi$ meson at nuclear matter density $\rho_{0}$ as a function of $\sigma_{N s}=m_{s}\langle N|\bar{s} s| N\rangle$. For both plots, the peak positions are given relative to the $\phi$ mass in vacuum.

be fitted by a constant plus a term linear in $\sigma_{S N}$ :

$$
\frac{m_{\phi}(\rho)}{m_{\phi}(0)}-1=\left[b_{0}-b_{1}\left(\frac{\sigma_{s N}}{1 \mathrm{MeV}}\right)\right] \frac{\rho}{\rho_{0}},
$$

$\rho_{0}$ representing the normal nuclear matter density. Our fit gives $b_{0}=(1.00 \pm 0.34) \cdot 10^{-2}$ and $b_{1}=(2.86 \pm 0.48)$. $10^{-4}$, which means that the mass shift changes its sign at a $\sigma_{s N} / 1 \mathrm{MeV}$ value of $b_{0} / b_{1}=34.9 \pm 13.1$. Using the variable $y$ instead of $\sigma_{s N}$, we get $0.174 \pm 0.040$ for the slope parameter (which corresponds to $C / y$ in [22], where a value of $0.15 \pm 0.045$ was obtained) with the sign of the mass shift switching at $y=(5.74 \pm 2.34) \cdot 10^{-2}$.

\section{DISCUSSION}

Let us try to understand the result of Fig. 4 4 by looking at the OPE of Eqs. (10 12) a bit more closely. From our discussion of the OPE in vacuum [Eqs. (7)9], see also Fig. 1), we know that the properties of the $\phi$ meson are essentially determined by the OPE terms up to dimension four. As the dimensionzero and -two terms do not have any density dependence, one 
can therefore expect that the finite density contributions to the dimension-four terms will control the modification of the $\phi$ peak. These terms of linear order in $\rho$ have the following form:

$$
\begin{aligned}
& -\frac{2}{27}\left(1+\frac{7}{6} \frac{\alpha_{s}}{\pi}\right) M_{N}+\frac{56}{27} m_{s}\left(1+\frac{61}{168} \frac{\alpha_{s}}{\pi}\right)\langle N|\bar{s} s| N\rangle \\
& +\frac{4}{27} m_{q}\left(1+\frac{7}{6} \frac{\alpha_{s}}{\pi}\right)\langle N|\bar{q} q| N\rangle+\left(1-\frac{5}{9} \frac{\alpha_{s}}{\pi}\right) A_{2}^{s} M_{N} \\
& -\frac{7}{12} \frac{\alpha_{s}}{\pi} A_{2}^{g} M_{N} .
\end{aligned}
$$

Written down numerically, this gives:

$$
\begin{aligned}
& -82.5 \mathrm{MeV}+2.19 \sigma_{s N} \\
& +3.95 \mathrm{MeV}+37.7 \mathrm{MeV}-31.3 \mathrm{MeV} \\
& =2.19\left[\left(\frac{\sigma_{s N}}{1 \mathrm{MeV}}\right)-32.9\right] \mathrm{MeV}
\end{aligned}
$$

Here, a positive coefficient results in a negative mass shift and vice versa. Therefore it is easily understood from the numbers above that for small values of $\sigma_{S N}$ a positive mass shift is observed, which turns into a negative one at $\sigma_{s N}=32.9 \mathrm{MeV}$. This quite accurately describes the situation observed in Fig. 4 and shows that it is indeed the dimension-four terms that almost completely determine the $\phi$ meson mass shift at finite density.

Another important point that needs some discussion is how our results can be understood in the context of the previous experimental studies on the $\phi$ meson at finite density. The E325 experiment at KEK has observed a significant excess on the lower mass side of the dilepton spectrum of slowly moving $\phi$ mesons produced in $12 \mathrm{GeV} p+A$ reactions, and extracted a negative mass shift of the $\phi$ of $35 \pm 7 \mathrm{MeV}$ at nuclear matter density [36]. In view of the results given in this paper, this finding is somewhat puzzling as such a large negative mass shift would correspond to values of $\sigma_{s N}$ larger than $100 \mathrm{MeV}$ (see Fig. 4), which seems to be in contradiction with recent lattice data [4-20], which suggest that $\sigma_{S N}$ should at least be smaller than $70 \mathrm{MeV}$. Furthermore let us mention here that according to [29], finite momentum effects will at the upper boundary of the lowest momentum bin of the E325 experiment $(\beta \gamma<1.25)$ lead to a further positive mass shift of 3 $\mathrm{MeV}$ for the transverse and $7 \mathrm{MeV}$ for the longitudinal component of the $\phi$ meson, and hence are not expected to help resolve the above discrepancy. On the contrary, they even lead to a further increase of the mass and therefore presumably will only worsen the situation.

The E16 experiment, to be performed at the J-PARC facility, will measure the $\phi$ meson in nuclear matter with much better statistics than in E325 and will thus hopefully provide much more precise information on the modification of the $\phi$ meson spectrum [65]. First of all, it will certainly be very interesting to see whether the result of the E325 experiment can be reproduced and what mass shift value will be extracted from the experimental data.

Related to the above topic, it is of course important to ask whether the sum rule approach could be missing some important effects and thus not be accurate enough to make precise statements on the behavior of the $\phi$ meson spectrum at finite density. Here, we want to stress once again that the OPE of this channel is relatively well determined because all important terms appear at dimension four or lower. This, however, of course does not fully exclude the possibility of some so-far neglected contributions quantitatively modifying our results in some way. Such higher order terms include condensates of higher dimension, further $\alpha_{s}$ corrections and terms beyond the linear density approximation. Among these, the terms of higher order in $\rho$ are presumably the most dangerous ones, as it is for instance known from in-medium chiral perturbation theory that the light quark condensate and finite density deviates about $5-7 \%$ from the linear behavior [66, 67]. Such a deviation could also exist for the strange quark condensate, which would modify our results accordingly. Therefore, for making our conclusions more solid, it would be desirable to take such kinds of contributions into account. We are planing to tackle this task in a future publication.

\section{CONCLUSION}

We have studied the behavior of the $\phi$ meson in cold $(\mathrm{T}=0)$ matter of finite baryonic density. This has been done with the help of a QCD sum rule approach, in which the finite density effects can be treated as modifications of the condensates of the QCD ground state, and thus provides a relation between the conversion of the $\phi$ meson spectrum and the change of the various order parameters of QCD. We have pointed out that, in the case of the $\phi$ meson, there is a strong correlation between the mass shift and the strange sigma term $\sigma_{S N}$ (shown in Fig. 4). This correlation emerges because of the specific properties of the $\phi$ meson channel. First, it is known that the $\phi$ meson remains relatively narrow even at nuclear matter density [35] and its broadening thus does not introduce a large uncertainty into the calculation. We have checked this point explicitly using a mock data MEM analysis and found that this uncertainty is only as large as a few $\mathrm{MeV}$ in terms of the $\phi$ meson mass shift (see Fig. 2). Second, the density dependence of the relevant terms of the OPE is well understood, the only unknown parameter being $\sigma_{S N}$, and the uncertainty due to not well constrained higher order contributions (such as the four-quark condensates) is sufficiently small.

Some more work still remains to be done in the future. As a first point, it would be important to test the stability of our results by including more higher order terms, especially those that go beyond leading order in density. It might also be possible to improve the MEM extraction of the spectral function with a recently proposed formulation of QCD sum rules on the complex Borel plane [68]. Furthermore, it would be interesting to confirm the earlier works on the $\phi$ spectrum at finite momentum [29, 30], to study the constraints provided by the sum rules on the finite momentum spectrum in detail, and to make predictions for the experimental measurements planned to be performed in the E16 experiment at J-PARC, at which the $\phi$ spectrum at both zero and finite three-momentum will be measured [65]. 


\section{ACKNOWLEDGEMENTS}

The authors thank Dr. S. Yokkaichi for motivating us to start this work and Professor M. Oka for enlightening discus- sions on the issues discussed in this paper. K.O. gratefully acknowledges the support by the Japan Society for the Promotion of Science for Young Scientists (Contract No. 25.6520). This work was supported by RIKEN Foreign Postdoctoral Researcher Program and the RIKEN iTHES project.
[1] X.-D. Ji, Phys. Rev. Lett. 74, 1071 (1995).

[2] A. Bottino, F. Donato, N. Fornengo and S. Scopel, Astropart. Phys. 18, 205 (2002).

[3] J. Ellis, K.A. Olive and C. Savage, Phys. Rev. D 77, 065026 (2008).

[4] R.D. Young and A.W. Thomas, Phys. Rev. D 81, 014503 (2010).

[5] R. Babich et al., Phys. Rev. D 85, 054510 (2012).

[6] S. Durr et al., Phys. Rev. D 85, 014509 (2012).

[7] R. Horsley et al. (QCDSF-UKQCD Collaboration), Phys. Rev. D 85, 034506 (2012).

[8] G.S. Bali, Phys. Rev. D 85, 054502 (2012).

[9] A. Semke and M.F.M. Lutz, Phys. Lett. B717, 242 (2012).

[10] W. Freeman and D. Toussaint (MILC Collaboration), Phys. Rev. D 88, 054503 (2013).

[11] P.E. Shanahan, A.W. Thomas and R.D. Young, Phys. Rev. D 87, 074503 (2013).

[12] H. Ohki et al. (JLQCD Collaboration), Phys. Rev. D 87, 034509 (2013).

[13] J.M. Alarcon, J. Martin Camalich and J.A. Oller, Phys. Lett. B730, 342 (2014).

[14] M. Engelhardt, Phys. Rev. D 86, 114510 (2012).

[15] P. Junnarkar and A. Walker-Loud, Phys. Rev. D 87, 114510 (2013).

[16] C. Jung (RBC and UKQCD Collaborations), PoS LATTICE 2012, 164 (2013).

[17] M. Gong et al. ( $\chi$ QCD Collaboration), Phys. Rev. D 88, 014503 (2013).

[18] C. Alexandrou et al. (ETM Collaboration), arXiv:1309.7768 [hep-lat].

[19] M.F.M. Lutz, R. Bavontaweepanya, C. Kobdaj and K. Schwarz, Phys. Rev. D 90, 054505 (2014).

[20] X.-L. Ren, L.S. Geng and J. Meng, arXiv:1404.4799 [hep-ph].

[21] P. Gubler and M. Oka, Prog. Thor. Phys. 124, 995 (2010).

[22] T. Hatsuda and S.H. Lee, Phys. Rev. C 46, 34 (1992).

[23] M. Asakawa and C.M. Ko, Nucl. Phys. A572, 732 (1994).

[24] Y. Koike, Phys. Rev. C 51, 1488 (1995).

[25] X. Jin and D.B. Leinweber, Phys. Rev. C 52, 3344 (1995).

[26] T. Hatsuda, S.H. Lee and H. Shiomi, Phys. Rev. C 52, 3364 (1995).

[27] F. Klingl, N. Kaiser and W. Weise, Nucl. Phys. A624, 527 (1997).

[28] S. Leupold, W. Peters and U. Mosel, Nucl. Phys. A628, 311 (1998).

[29] S.H. Lee, Phys. Rev. C 57, 927 (1998).

[30] S. Leupold and U. Mosel, Phys. Rev. C 58, 2939 (1998).

[31] S. Zschocke, O.P. Pavlenko and B. Kämpfer, Eur. Phys. J. A 15, 529 (2002).

[32] B. Kämpfer, O.P. Pavlenko and S. Zschocke, Eur. Phys. J. A 17, 83 (2003).

[33] R.S. Hayano and T. Hatsuda, Rev. Mod. Phys. 82, 2949 (2010).

[34] S. Leupold, V. Metag and U. Mosel, Int. J. Mod. Phys. E 19, 147 (2010).

[35] A. Polyanskiy et al., Phys. Lett. B 695, 74 (2011).
[36] R. Muto et al., Phys. Rev. Lett. 98, 042501 (2007).

[37] M.A. Shifman, A.I. Vainshtein and V.I. Zakharov, Nucl. Phys. B147, 385 (1979); B147, 448 (1979).

[38] C. Gale and J. Kapusta, Nucl. Phys. B357, 65 (1991).

[39] S.C. Generalis and D.J. Broadhurst, Phys. Lett. B139, 85 (1984).

[40] K.G. Chetryrkin, S.G. Gorishny and V.P. Spiridonov, Phys. Lett. 160B, 149 (1985).

[41] G.T. Loladze, L.R. Surguladze and F.V. Tkachov, Phys. Lett. 162B, 363 (1985).

[42] L. Surguladze and M. Samuel, Rev. Mod. Phys. 68, 259 (1996).

[43] S. Bethke, Eur. Phys. J. C 64, 689 (2009).

[44] P. Colangelo and A. Khodjamirian, At the Frontier of Particle Physics/ Handbook of QCD (World Scientific, Singapore, 2001), Volume 3, 1495.

[45] J. Beringer et al. (Particle Data Group), Phys. Rev. D 86, 010001 (2012).

[46] C.A. Dominguez, N.F. Nasrallah and K. Schilcher, JHEP 02, 072 (2008).

[47] Y. Aoki et al., Phys. Rev. D 83, 074508 (2011).

[48] S. Choi, T. Hatsuda, Y. Koike and S.H. Lee, Phys. Lett. B312, 351 (1993).

[49] J. Gasser, H. Leutwyler and M. Sainio, Phys. Lett. B253, 252 (1991).

[50] X. Jin, T.D. Cohen, R.J. Furnstahl and D.K. Griegel, Phys. Rev. C 47, 2882 (1993).

[51] A.D. Martin, W.J. Stirling, R.S. Thorne and G. Watt, Eur. Phys. J. C 63, 189 (2009).

[52] E.G. Drukarev and E.M. Levin, Prog. Part. Nucl. Phys. 27, 77 (1991).

[53] T.D. Cohen, R.J. Furnstahl and D.K. Griegel, Phys. Rev. C 45, 1881 (1992).

[54] K.G. Chetryrkin, S.G. Gorishny and F.V. Tkachov, Phys. Lett. B119, 407 (1982).

[55] S. Zschocke, T. Hilger and B. Kämpfer, Eur. Phys. J. A 47, 151 (2011).

[56] P. Gubler, A Bayesian Analysis of QCD Sum Rules, Springer Theses, Springer Japan, 2013.

[57] M. Jarrell and J.E. Gubernatis, Phys. Rep. 269, 133 (1996).

[58] M. Asakawa, T. Hatsuda, and Y. Nakahara, Prog. Part. Nucl. Phys. 46, 459 (2001).

[59] E.V. Shuryak, Rev. Mod. Phys. 65, 1 (1993).

[60] T. Waas, N. Kaiser and W. Weise, Phys. Lett. B379, 34 (1996).

[61] L. Tolos, D. Cabrera and A. Ramos, Phys. Rev. C 78, 045205 (2008).

[62] F. Klingl, T. Waas and W. Weise, Phys. Lett. B431, 254 (1998).

[63] E. Oset and A. Ramos, Nucl. Phys. A679, 616 (2001).

[64] D. Cabrera and M.J. Vicente Vacas, Phys. Rev. C 67, 045203 (2003).

[65] D. Kawama et al., PoS, Hadron 2013, 178 (2014).

[66] N. Kaiser, P. de Homont and W. Weise, Phys. Rev. C 77, 025204 (2008).

[67] S. Goda and D. Jido, Phys. Rev. C 88, 065204 (2013). 
[68] K.-J. Araki, K. Ohtani, P. Gubler and M. Oka, Prog. Theor. Exp. Phys. 2014, 073B03. 